\title{
Involvement of Learners' Characteristics within the Allocation of Submissions in the context of Peer Assessment in MOOCs
}

\author{
M. A. Abrache \\ IRF-SIC Laboratory, FSA \\ Ibn Zohr University \\ Agadir, Morocco
}

\author{
A. Qazdar \\ GMES Laboratory, ENSA \\ Ibn Zohr University \\ Agadir, Morocco
}

\author{
C. Cherkaoui \\ IRF-SIC Laboratory, ENCG \\ Ibn Zohr University \\ Agadir, Morocco
}

\begin{abstract}
The contribution in the context of this paper relates to the stage of allocating submissions to the assessors within the peer assessment process in MOOCs. We propose an algorithm for the distribution of assignments that involves the learner characteristics related to the evaluation in the methodology of allocating assignments. The inputs of this algorithm are the assessment profiles of learners which include their basic characteristics linked to the evaluation process. The creation of these profiles stands on the use of an assessor model inspired from the literature on learners modeling, a model that we are also discussing in this paper.
\end{abstract}

\section{General Terms}

E-learning, online education, student assessment, algorithms, learners modeling.

\section{Keywords}

Peer assessment, peer review, learner profile, assessor model, MOOC, online assessment tools.

\section{INTRODUCTION}

Massive Open Online Courses (MOOCs) are online courses that have been organized according to a schedule with deadlines, tests, activities, homework, exams, and online communication forums for students [1]. They offer to a massive number of students an opportunity to benefit from an open-access free or at a reduced price higher education regardless of their locations or backgrounds [2].

The massive nature of these courses implies a number of challenges to maintain the engagement of students within the teaching-learning-evaluation/feedback process [3]. Among these challenges there is the reliability of assessment and the problem of the drop-out of learners [4].

In this paper, we focus on the assessment component in MOOCs which represents a topic of concern to researchers in the field of online learning environments.

A concern that is reflected in the proposal of assessment approaches that target more reliability and more validity, combined with the objective of ensuring effective feedback to learners [5].

In online learning environments, peer assessment is one of the methods used to address the assessment issue. This method can take a part on the realization of the modern objectives of assessment that differs from those of classical academic education [6]. Indeed, the task of memorizing and assimilating basic knowledge is no longer the central issue, but rather the development of cognitive skills such as problem solving, critical thinking and metacognitive skills such as selfreflection and self-assessment, as well as other social skills such as the ability to engage in discussions, the capacity to persuade, and working in groups.

For Dochy [7], assessment refers not only to the measurement of assimilated information, but also to the students' participation, their application of knowledge and skills, their integration into the learning environment, and as well to their construction of knowledge instead of its reproduction. This implies the need of involving the personal characteristics of learners in the application of peer assessment, especially in online learning environments where the assessment is more learner-centered.

The peer assessment process can be summarized as follows:

- Position of the questions of evaluation and specification of the rubric.

- Submission of the homework by learners.

- Allocation of the submissions to the participants in order to be assessed.

- Peers grading exercise and the writing of feedback.

- Calculation of the final score.

- Contact peer assessment score and feedback to each learner.

The openness of MOOCs implies dealing with enrolled learners who are not true peers, because of their different backgrounds, abilities, objectives, and behavior patterns [5].

The interest in this paper is related to how involve the assessors' characteristics into preserving the reliability and the credibility of assessment within the scope of submissions' allocation stage of the peer assessment process in MOOCs.

The remainder of the present paper is organized as follows: the section 2 is a brief overview of the literature related to the assessment within the field of online learning environments, especially the platforms integrating peer assessment in MOOCs. The section 3 is a critic over the solutions mentioned in the overview. Within the section 4 , we present an assessor model that stands on the assessment profiles of learners; while in the fifth, we introduce the algorithm of allocating submissions. The section 6 describes the simulation of the solution and exposes its results. Finally, the last section is a discussion of the results with a conclusion and perspectives.

\section{BACKGROUND}

Automatic correction techniques are commonly used in many MOOC platforms according to various forms as multiplechoice, short answer questions, computer code, and vocabulary activities [8]. 
However, these solutions showed limitations in their ability to assess and give feedback on project tests, open-ended exercises, or essays [9].

Moreover, Edx MOOC platform has tried AI grading using Automated Essay Scoring (AES). AES stands on computer programs based on natural language processing (NLP) algorithms and machine learning techniques. It aims to attribute grades to essays written by learners [8].

Yet, there are limitations in the automatic classification algorithm, which cannot handle tasks that do not correspond to the training examples [10], and fails to capture the semantic meaning from learners' answers, so the ability to provide constructive comments is negatively affected [11].

The use of peer assessment as a solution based on the intelligence of the crowd was then a necessity [4]. A solution that promotes learner autonomy and responsibility and helps in the transfer of responsibility of assessment from the teacher to the student.

Topping has defineds the peer assessment as "an arrangement in which individuals consider the amount, level, value, worth, quality or success of the products or outcomes of learning of peers of similar status" [12].

This form of assessment plays an important role in ensuring formative evaluation and feedback on learners' performance, and allows students to improve their knowledge by evaluating peers works [13].

In a large-scale class such as MOOCs, where the burden of grading is too high, we are witnessing a greater reliance on peer assessment which has become a viable method widely used in these online classes [3].

The use of peer assessment offers promising solutions to assess complex tasks being carried out by thousands of students, to help them improve their capacities and their understanding [14] and to engage them in the learning process [15]. Indeed, peer-based assessment makes MOOCs more interactive and participates on improving the completion rates among the enrolled learners [16].

However, to apply to peer assessment in MOOCs classes, a problem of validity is generally encountered, because of the number of learners who are still in the accumulation of knowledge and who do not yet have sufficient knowledge and capacity to assess peers' assignments with high accuracy.

Meanwhile, there are other personal factors that impacts the assessment process, namely: social styles [17], learners' attitude towards the assessment [18], skills, prejudices, culture, preferences, experiences, abilities, and learning styles [19].

Many online peer review/assessment tools were proposed in the literature to deal with this concern such as Aropä [20], SWoRD [21], Moodle Workshop module [22], Web-SPA [23], CeLS [24], PeerScholar [25], etc. We give within the rest of this section an overview of some principal online peer review tools, then we show the features of the main implementations of peer assessment in MOOCs contexts and essentially their mechanisms of assigning submissions to participants.

\subsection{Online peer review tools}

\subsubsection{Aropä}

Sluijsmans et al. (2001) noticed that learners do not tend to give marks and are more inclined to just provide feedback
[26]. In this sense, Aropä which is a web-based tool that has been first created in 2007 for an academic use, integrates a set of peer assessment activities. The development of Aropä has followed a design that favors peer feedback to the detriment of peers grading [27]. It uses an algorithm that compares the similarity between the feedback of reviewers in order to achieve more accuracy. Aropä allows instructors to specify the number of reviews to be drafted by learners, which may possibly include a self-review. The allocation of submissions is randomly made to ensure a just distribution according to the amount of feedback indicated by the instructor [20].

\subsubsection{SWoRD}

Scaffolded Writing and Rewriting in the Discipline is a webbased client-server peer-review application that has been developed to support students writing practices. It works on both peer assessment and feedback generation. As for Aropä, the allocation of assignments occurs at random once all students have submitted their works. SWORD's reviewing is based on a three-dimensional model that consists of flow, logic and insight. To give more accuracy to the feedback, this system uses three precision indices to calibrate the inaccuracy of students' reviews which are: systemic differences, consistency and propagation [21]. SWoRD provides a support to learners in the effective review phase through a form of task specified feedback prompts and grading rubrics [28].

\subsubsection{The Moodle Workshop}

The Moodle Workshop module for formative peer assessment is a collaborative grading system that allows students to assess each other's projects respecting different grading strategies specified by the instructors; these strategies are: ungraded, peer-graded, instructor-graded, or a combination of peer- and instructor-graded strategies. It gives instructors the possibility to edit peer assessment settings to manage the assessment criteria, or to supply some pre-evaluated samples for students to test their assessment ability, or to custom the feedback providing mechanisms, etc. The allocation of submissions in this tool is done either manually or randomly [22].

\subsubsection{Correction of Evaluation Results}

Within the field of the correction of evaluation results, some proposed tools consider the personal characteristics of the assessors. These tools measure a number of indices such as the Reviewer Competency Index (RCI) within Coursera platform using the Calibrated Peer Review (CPR) [9], or the Credibility Index (CI) [29], which evaluates the levels of inaccuracy, incompatibility and non-transferability of assessments among learners.

The models of Piech [9] and Goldin [30] introduce unobservable estimated parameters as the prejudices of the assessors, whereas Lan et al. [19] focus on the learning styles.

\subsection{Peer Assessment in MOOCs}

Coursera which is one of the main MOOCs' classes providers, introduced the Calibrated Peer Review (CPR) to calibrate peers' reviews within the context of peer assessment process. The CPR includes taking a random sample of assignments deposited by the participants, these assignments are evaluated by the staff, and randomly distributed to the assessors simultaneously with other assignments not included in the sample [9].

Moreover, within the Edx platform, learners are asked to assess some pre-evaluated assignments. Learners obtain the authorization to evaluate peers' submissions once they manage to assign similar marks compared with those given by the 
instructors. Edx's documentation encourages instructors to request for each assignment a number of assessments beyond of what they believe is necessary. This has been suggested to overcome the situation where a participant did not assess herlhis assigned submissions [31].

Besides, the principle of submission priority [32] represents a mechanism that aims to balance the distribution of assignments and their possibility to be examined by different assessors at the same time. This principle characterizes the submissions by a priority and a minimum necessary number of assessments to be ensured. It is a mechanism of distributing the assignments based in their order of priority. Once the allocation of an assignment is done, its priority is decreased as well as the number of necessary assessments. Six hours later, if the assignment has been effectively evaluated, the priority of the submission belonging to the participant who did the assessment is increased. This principle rewards assessors who carried out the review of their assigned submissions by ensuring that their assignments would be assessed with an upper priority. Otherwise, if the assignment has not been evaluated within the six hours, its priority and the number of necessary evaluations increases automatically so that it will be redistributed a new time.

\section{Critics Over the Proposed Solutions}

Except for the principle of submission priority, most of the work carried out in the context of peer assessment does not usually provide sufficient detail on the task of assigning submissions to assessors, or simply tend to use a randomized method to perform this task. This is the case for the aforementioned online peer review tools that allocate the submissions randomly, or for the CPR that is limited to calibrating the assessments and is not intended to exploit the allocation of assignments in optimizing the whole process.

Furthermore, the criticism over the tools used in the field of correcting evaluation results lies in the fact that they do not interfere sufficiently with the allocation of submissions, and that they only apply at a level subsequent to what we are trying to optimize.

The optimization of peer assessment process requires naturally optimizing each of its steps. However; the focus on the allocation of submissions step all over the tools and platforms mentioned above is related only to a technical aspect which is overcoming the problem of the not assessed submissions and does not aim to optimize the process of assessment based on the distribution of assignments. While the introduction of assignment competency of learners as a parameter to be considered in the allocation of submissions may offer a potential capacity for optimizing the process of peer assessment

For example, it is possible that a submission is evaluated by a group of learners made up entirely of novice assessors in terms of their evaluation competency. This situation, on the one hand, affects negatively the reliability and the accuracy of this evaluation, and, on the other, calls into question the credibility of the entire peer assessment process. Especially, as it is possible that another assignment may be assessed by learners with much more advanced capacities than the latter group, which implies that the evaluation conditions are not equal for each assignment and may have an impact on the motivation of learners not only for this form of assessment but for the course in general.
Dealing with an assessment profile relying on an assessor model is a fundamental element in the direction of gathering information about the personal parameters that may influence the learner assessment capacity within the context of MOOCS or other online learning tools.

\section{ASSESSOR MODEL}

Online learning platforms like MOOCs allow learners to have a lot of autonomy, to be responsible for their own learning progress, and to be able to evaluate their advancement as well as their learning outcomes.

The optimization of the peer assessment process includes, in a large part, the enumeration of the personal characters of the assessor profile in order to have an idea about the overall characteristics of the community that operates the assessment.

From this perspective, it seems necessary to create an assessor model that naturally inherits models and standards of learners modeling such as the "PAPI Learner" standard [33], or IMS LIP (Information Management System - Learning Information Package) [34] or the model proposed by Battou in the context of the ALS-CPL project.

Battou [35] summarized the various definitions of the learner modeling as a process which covers the life cycle of the setting up of a learner model.

The process of the learner modeling includes the acquisition of knowledge about the learner, and then constructing, updating, maintaining and exploiting this model.

In the same way, the proposed assessor model can be defined as a representation of the assessor which includes all information and characteristics intrinsic to the evaluation process. In this context, this evaluation is a peer assessment within a MOOC context.

This assessor model (see Figure 1) follows a qualitative approach for the representation of information and allows the characterization of each assessor according to various factors:

- The ability to interact with the MOOC platform, and mainly with the evaluation modules.

- The expertise related to the course domain.

- The previous assessment expertise.

- The effective competence of assessment.

On the other hand, there are other characteristics that may have a significant influence on learners' ability to assess as their learning styles.

\subsection{Interaction capabilities with the platform}

ICT competence and the language proficiency are two parameters that affect the interaction of learners with the MOOC platform. Learners must have some basic skills, including computer exploitation capabilities and a good mastering of the language used in the course [36]

This has become more vital for the assessment experience that requires a sufficient understanding of the subject and the assignments of peers as a means of making an appropriate judgment. Language proficiency is also reflected in feedback writing. 


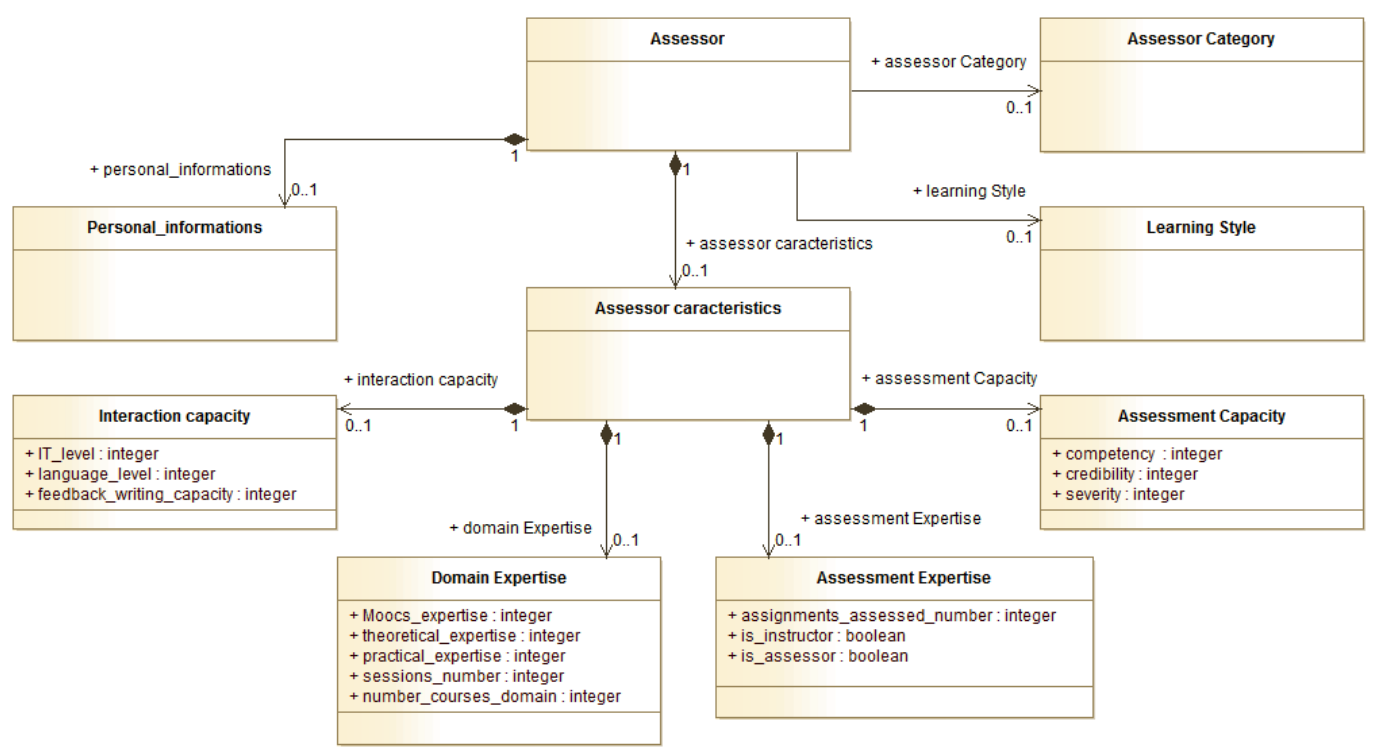

Fig 1: Assessor Model

Resistance to peer feedback is rare, compared to the relatively more frequent resistance to the formal peer assessment [37], which means that a good quality of feedback can be an asset to ensure acceptance of the assessment results.

\subsection{Expertise within the course's domain}

Antecedent expertise related to the course's domain is a factor that influences not only the ability to achieve learning objectives, but also the capacity to conduct a reliable assessment for peers' works. This domain expertise can be divided into three principal aspects: the previous experiences in MOOCs platform, the theoretical competence that underlies the course field, and the practical competence linked to the application know-how. In addition, other factors influence the domain's expertise such as the number of previous sessions of the course followed by the assessor, as well as the courses related to the same subject that have been previously completed.

\subsection{Assessment Expertise}

The experience concerning the assessment exercise is an important factor influencing the effectiveness of evaluation. Having an ability to interact with the platform or some antecedent knowledge of the domain is not always sufficient. The methodology and the competence of assessing enable more accurate identification of the mistakes and the gaps in peers' works, which is expressed in the relevance and the clarity of the feedback.

This assessment expertise can be estimated according to the number of assignments corrected previously, as well as through the nature of the assessor's job. A teacher tends to have more assessment expertise, and so for an assessor carrying out a profession that involves making evaluations, and writing reports (feedbacks).

\subsection{Assessment capacity}

As we illustrated before, measuring learners' ability to assess is widely used in the field of the correction of peer assessment evaluation results.

Some other models use this measurement as the multifaceted Rasch measurement model (FACETS) proposed by Linacre (1989) consists of three facets: the student competencies, the difficulty of the field or the assignment, and the differences between the severity of assessors [38].

The assessor model integrates three basic parameters that may represent globally the assessment capacity: the competency, the credibility, and the severity of the assessor.

\subsection{Learning style}

Lan, Graf and Lai [19] relied on the Felder-Silverman Learning Style Model (FSLSM) [39] to give a weight to each learner's submitted score for each assessment criteria according to herlhis learning style. The FSLSM has divided students' learning styles into four dimensions: active/reflective, sensory/intuitive, visual/verbal and sequential/global. Lan, Graf and Lai specified an assessment weighting for all the criteria according to the learning style of students.

For example, in the context of a home work having as subjects: the development of a website, they gave a great weight to students with an active learning style for all the assessment criteria as the learners of this category tend to try things and prefer to learn by communicating and collaborating with other pairs, which is not the case for learners with a reflective style. For students with a visual learning style, they seem to be able to give a more accurate assessment for the "Site Structure" criterion, as well as for the "Graphs" criterion that deals with the assessment of graphs on the website. For verbal learners, they have more weight regarding the criterion "Content." For each remaining criterion, a weight for the student assessment has been specified according to their learning style.

It is possible to use the same principle to create a database containing all the evaluation criteria belonging to a particular domain with a specification of the evaluation weights for each criterion based on the different learning styles of learners. In addition, the use of an evaluation that stands on different evaluation criteria may provide more reliability and precision for the evaluation result. 


\section{ALLOCATING SUBMISSIONS ALGORITHM}

The involvement of learner assessment capacity in the allocation of submissions can have a positive influence on the accuracy and reliability of peer assessment. Our proposal is to dispatch each assignment to a group of assessors made up of members from different categories of learners.

These categories are created according to the capacities of assessment of the participants, so that each category contains assessors with a convergent level, which allows learners to have almost equal chances for their work to be evaluated under the same conditions and can lead to a gain of the credibility of assessment.

The assessor model mentioned above represents the characteristics related to the learner assessment capacities. Based on this model, an "assessment score" is measured according to these characteristics in order to distinguish the overall evaluation capacity of each participant. The learners are asked to specify on a scale of five an appropriate score for herlhis level of competence linked to every characteristic mentioned in the assessor model, so they can give an elementary score for each characteristic affecting their capacity of assessment.

Since the influence of each characteristic on the overall assessment capacity of the learner differs from one characteristic to another, we introduced for each characteristic a "coefficient of influence" corresponding to its influence on the assessment capacity. For instance, because of the importance of the assessment experience, this characteristic corresponds to a higher coefficient of influence, so that the assessment score depends more on this characteristic compared to the others.

The same logic was followed for the other competencies having a significant impact on the assessment capacity. The sum of the elementary scores multiplied by the influence coefficients gives the overall assessment score $\widehat{S}_{l}$.

This score $\widehat{S}_{l}$ of the assessor $i$ is calculated through the following formula:

$\widehat{S_{l}}=C^{T} \times S_{i}=\sum_{j=1}^{n} C_{j} \times S_{i j}$

where $n$ is the number of the assessor's characteristics,

$S_{i}=\left[\begin{array}{c}S_{i 1} \\ \vdots \\ S_{i n}\end{array}\right]$ the matrix of the elementary scores obtained by the assessor $i$ for each of the $n$ characteristics,

$C=\left[\begin{array}{c}C_{1} \\ \vdots \\ C_{n}\end{array}\right]$ the matrix of the coefficients of influence corresponding to each of the $n$ characteristics.

The assessors' scores are used for the categorization of assessors, which consists of grouping learners according to their assessing abilities. To do so, two approaches may be followed.

The first is to specify a threshold corresponding to each category; this implies more reliability, but at the same time a greater charge on the assessors of the higher category, since the category of expert assessors will naturally have fewer members but more assignments to be evaluated. This situation may cause a negative impact on the motivation of the members of this category to exercise the assessment.
The second approach is to categorize the assessors in a way that relates to the overall level of competency. After having specified the number of categories, the number of assessors is divided on this number, and the quotient $q$ of this division is about the number of assessors per category. The category representing expert assessors will contain the first $q$ participants with the highest scores, then the advanced assessors' category will contain the following $q$ assessors in the order of score and so on for the other categories.

This approach allows a representation of all categories when dispatching the assignments and implies an equal burden for all assessors, without negatively influencing the motivation of learners.

We can summarize our submissions allocating algorithm in the following steps:

- Receiving assessor profiles of all participants.

- Classification of the assessors in four different categories according to their evaluator scores.

- Submission of the homework by learners.

- Verification of the evaluation charge of assessors before performing the allocation.

- Allocating submissions one by one to a member from each category.

\section{SIMULATION \& RESULTS}

\subsection{Acquisition of information}

Before describing the simulation, it is fundamental to explain the method used to create the assessor's profile. Participants are asked to complete a questionnaire that stands on the assessor's model in order to gather the information feeding the assessors' database. This questionnaire was created on the basis of a model engine called Twig used in the context of the Symfony framework.

Concerning the learning style, there are several examples of questionnaires related to this concept that may be found on the web, and which can be used to collect data about the learning styles of students.

(e.g. http://www.psychomedia.qc.ca/tests/index-of-styles-oflearning).

The questionnaire consists of four steps, each step collects information related to some of the aspects mentioned in the model above. Figure 2 shows step 2 of the questionnaire.

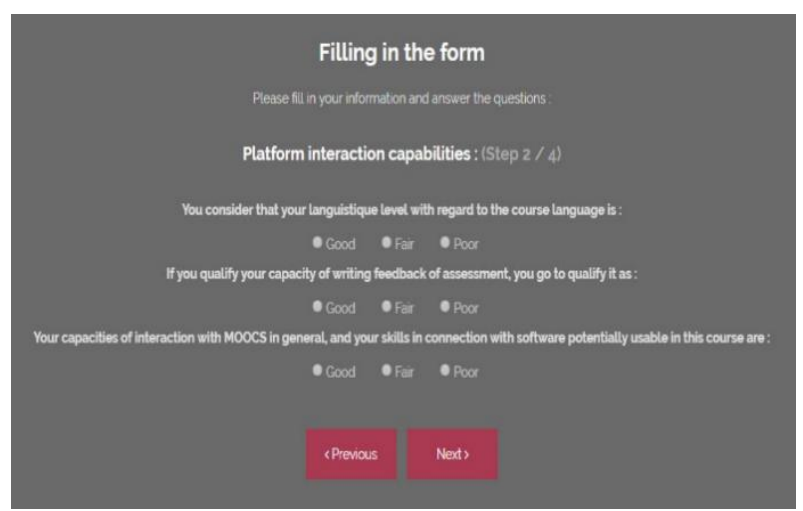

Fig 2: Assessor questionnaire

\subsection{Description of the Simulation}

To test the utility of the solution, we opted for an application to simulate the implementation of the algorithm as part of a MOOC course. To proceed, the application relies on an artificial agent using the JADE platform based on Java programming language [40]. 
The choice of JADE is mainly linked to the possibility of enriching the algorithm through the integration of some complementary aspects in the perspective of developing an assistant agent for peer assessment in MOOCs.

This simulation includes all the basic characteristics mentioned in the assessor model except for the learning style and the measurable capacities through the effective assessment experience within the course.

Our agent allocator of submissions has two behaviors, the first consists of receiving all the profiles of participants enrolled in the course, and then classifying these profiles into categories of assessors, while in the second behavior the agent performs the effective distribution of assessments to the categories' members.

\section{- Classification behavior:}

This classification is based on the assessor's score which is calculated using the information provided by the submitted form. After classifying the learners ordered by their scores, the assessors' community is divided into four categories: beginners, intermediates, advanced and experts. The classification considers ensuring the same number of assessors in each category.

\section{Distribution behavior:}

As mentioned earlier, it is the behavior containing the effective allocation of submissions to the learners. We assume that the distribution is made once a participant submits herlhis homework, which leads to develop a cyclic behavior in order to dispatch the assignment once is added to a member of each category. The execution of this behavior focuses on two priority checks: the first is that an assignment should not be assigned to its owner and the second is that in all categories each member should evaluate an equal number of submissions compared to the other assessors belonging to the same category.

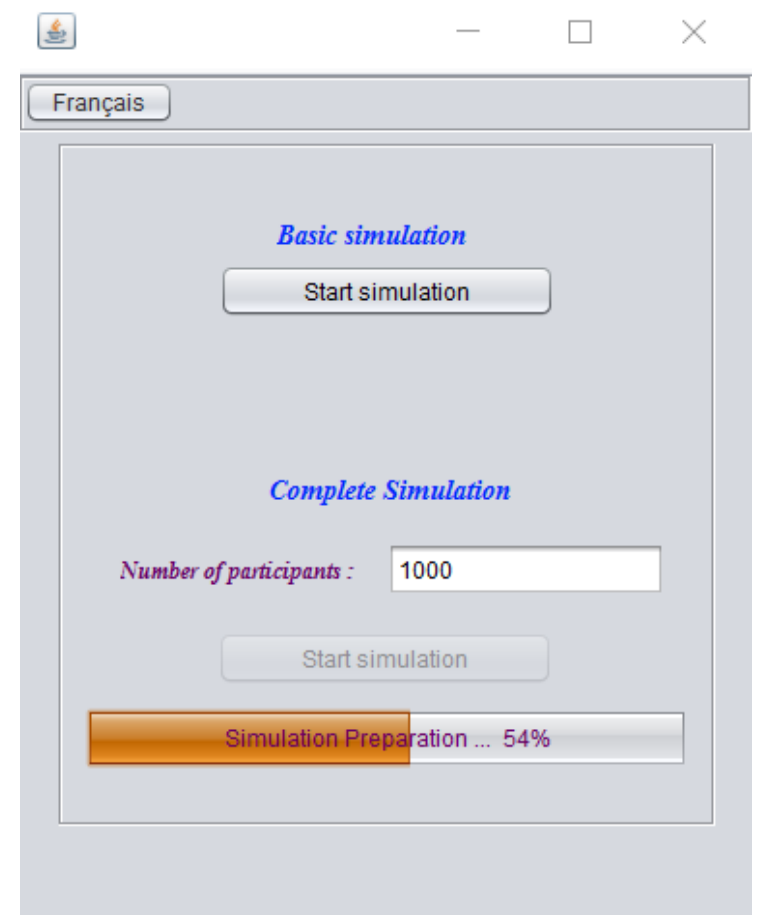

Fig 3: Simulation preparation interface

\subsection{Results of Simulation}

The simulation of the allocation of submissions algorithm is performed in three steps, the first consisted of specifying a number of participants and generating the data for the simulation. It can also use the assessor questionnaire to add assessors' profiles.

To visualize the results, we used an example of a course with 1000 participants for which a random generation of assessors' profiles is executed (see Figure 3). For the basic simulation, it includes a small number of assessors and allows to simulate the distribution of submissions one by one.

The second step of the simulation is the classification of the assessors by category. It is at this stage that the allocator agent starts the classification process of the participants and then renders the results to the same interface (see Figure 4).

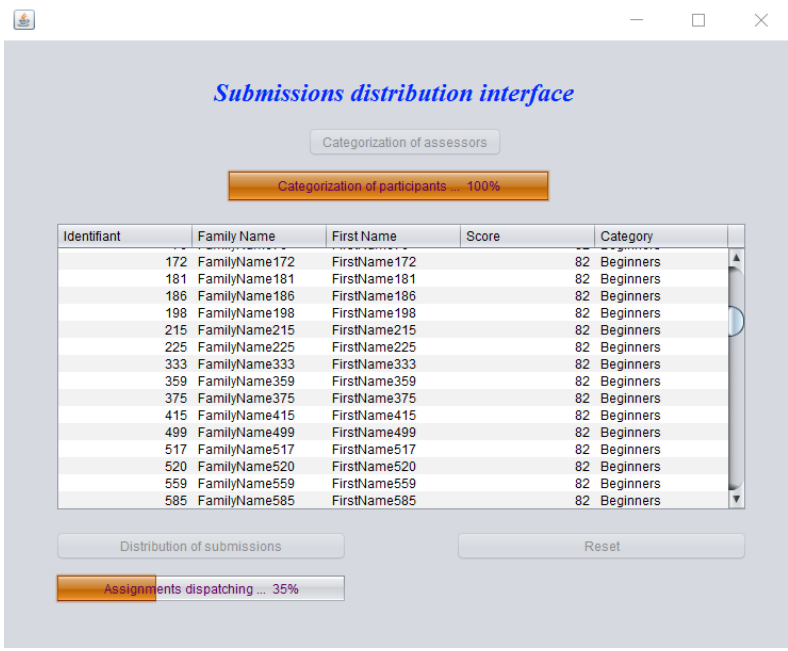

Fig 4: Submissions Distribution Interface

As indicated previously in the description of the algorithm, the specification of the members of each category does not use thresholds for the classification according to the assessor's score but rather seeks the same number of assessors per category based on these scores.

In the listed previous example, the number of evaluators per category is 250 . The top 250 evaluators according to the assessors scores belong to the experts' category, while the next 250 represent the advanced category, then the 250 that followed are the members of the intermediate category, and finally the last 250 constitute the beginners' category.

After viewing participants' information, their assessment scores and categories; the simulation proceed to the next step of the algorithm which is the effective allocation of submissions. 


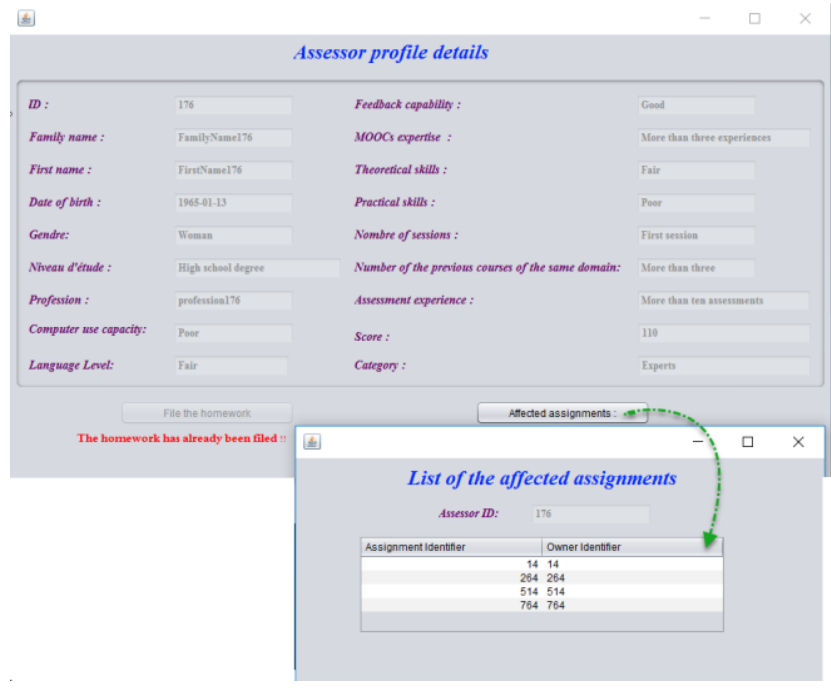

Fig 3: Interface of learner assessment profile

Simultaneously with the classification behavior, the distribution behavior is triggered. After the end of the classification process, the agent is waiting for the submission of a new homework by a participant to assign it to an assessor from each category.

After that a click on the simulation of the distribution button is performed, the filing of submission of assignments for each of learners is simulated. Once the agent realizes the existence of a not allocated assignment, it immediately performs the distribution process (see Figure 4).

Selecting an assessor from the list makes it possible to view his complete profile and gives access to his assigned submissions at the end of the distribution step. The figure 5 shows a participant's profile with an assessment score of 110 , who belongs to the category of "Experts," and who already filled his/her homework and has 4 assignments to evaluate.

The interface distribution of the assignments (see Figure 6) allows having an idea on the way which the assignments have been distributed.

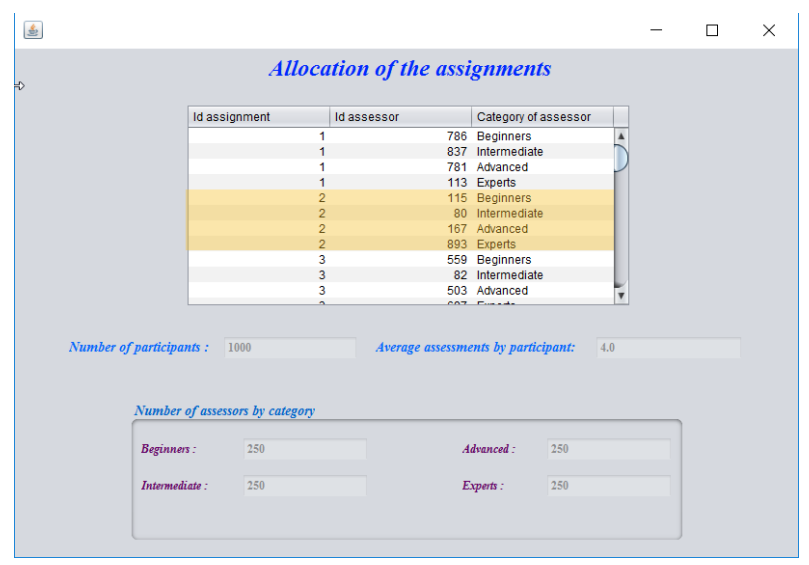

Fig 4: Interface of the allocation results

\section{DISCUSSION, CONCLUSION AND FUTURE WORK}

The results of the simulation of the algorithm are aligned with the first objective, which is to distribute the submissions so that every assignment is evaluated by a member of each category.
Similarly, the simulation showed a reasonable average of submissions (4 in the example) to be assessed per learner. This average represents a principal parameter in the fair distribution of assignments reflecting the correct execution of the distribution process.

Besides, it is important to mention that we considered the ideal case where all the learners have submitted their homework and mainly that they effectively evaluated all their assigned submissions.

Yet, the system may be in front of a situation where the learners did not evaluate all their assigned homework. The principle of priority of submission is an applicable mechanism in this system and which can be modified to consider the use of the categorization of assessors for the distribution in such situations.

This work introduced a first assessor model based on the personal characteristics of the learner. We intend to evolve the system standing on this model by considering the update of the assessor's profile, and including data related to the assessment capacity gathered through the effective experience of assessors.

Furthermore, we plan to measure the effect of this solution in a real situation to compare the reliability of the assessment's results regarding a random distribution of the assignments, and at the same time to be able to observe its effect on the motivation of the learners, and on the development of their capacity of assessment.

There are also a number of next steps we are investigating, as the implementation of some other elements of the model, especially the use of evaluation criteria in parallel with learning styles. The same for the assessment expertise of assessors, which must be updated as the evaluations carried out by the participants, and as regards to the measurement of the effective competence of assessment that depends on parameters such as the competency of the assessor, and the validity of his assessments, etc.

Integrating a feedback support module is one of the perspectives of our work because of the importance of this component in the success of the learning process, and thus the validity of the peer review.

The measurement of the assessment score that considers the characteristics of the course as the language used and the area of specialization, may be used in the pre-correction of evaluations by giving different weights to the assessment of each member of the group evaluating the same assessment according to the category to which he belongs.

The categorization of assessors gives the ability to offer adequate assistance to the assessors of every category, this assistance may be manifested through the way of explaining the rubric to the learners that may depend on their level as assessors, or through raising the awareness on the importance of writing constructive feedback.

Finally, the algorithm and its application represent the foundations towards a global purpose of our research work, which is the creation of a peer assessment assistance agent within the context of MOOCs. 


\section{REFERENCES}

[1] Siemens, G., Massive open online courses: Innovation in education. Open educational resources: Innovation, research and practice, 2013. 5.

[2] González, S.J., et al., MOOCs and Multiple Learning Styles. User-Centered Design Strategies for Massive Open Online Courses (MOOCs), 2016. 30

[3] Suen, H.K., Peer assessment for massive open online courses (MOOCs). The International Review of Research in Open and Distributed Learning, 2014. 15(3).

[4] Yousef, A.M.F., et al. The Impact of Rubric-Based Peer Assessment on Feedback Quality in Blended MOOCs. in International Conference on Computer Supported Education. 2015. Springer.

[5] Meek, S.E., L. Blakemore, and L. Marks, Is peer review an appropriate form of assessment in a MOOC? Student participation and performance in formative peer review. Assessment \& Evaluation in Higher Education, 2016: p. $1-14$.

[6] Lojacono, F.G., Les MOOC: du paradigme constructiviste à celui de la reliance MOOCs: From a constructivist approach to a reliant paradigm. Revista de Lenguas para Fines Específicos, 2016. 22: p. 12-30.

[7] Dochy, F., A new assessment era: different needs, new challenges. Learning and Instruction, 2001. 10: p. 11-20.

[8] Balfour, S.P., Assessing Writing in MOOCs: Automated Essay Scoring and Calibrated Peer Review (TM). Research \& Practice in Assessment, 2013. 8.

[9] Piech, C., et al., Tuned models of peer assessment in MOOCs. arXiv preprint arXiv:1307.2579, 2013.

[10] Mitros, P., et al. An integrated framework for the grading of freeform responses. in The Sixth Conference of MIT's Learning International Networks Consortium. 2013.

[11] Kulkarni, C., et al., Peer and self assessment in massive online classes, in Design thinking research. 2015, Springer. p. 131-168.

[12] Topping, K., Peer assessment between students in colleges and universities. Review of educational Research, 1998. 68(3): p. 249-276.

[13] Topping, K.J., Peer assessment. Theory into practice, 2009. 48(1): p. 20-27

[14] Admiraal, W., B. Huisman, and M. Van de Ven, Self-and peer assessment in massive open online courses. International Journal of Higher Education, 2014. 3(3): p. p119.

[15] Jiao, J., et al., Improving Learning in MOOCs Through Peer Feedback: How Is Learning Improved by Providing and Receiving Feedback? in Learning and Knowledge Analytics in Open Education. 2017, Springer. p. 69-87.

[16] Khalil, H. and M. Ebner. MOOCs completion rates and possible methods to improve retention-a literature review. in World Conference on Educational Multimedia, Hypermedia and Telecommunications. 2014.

[17] May, G.L. and L.E. Gueldenzoph, The effect of social style on peer evaluation ratings in project teams. Journal of Business Communication, 2006. 43(1): p. 4-20.
[18] Lin, S.S., E.Z.-F. Liu, and S.-M. Yuan, Web- based peer assessment: feedback for students with various thinking- styles. Journal of Computer Assisted Learning, 2001. 17(4): p. 420-432.

[19] Lan, C.-H., et al., Enrichment of peer assessment with agent negotiation. IEEE Transactions on Learning Technologies, 2011. 4(1): p. 35-46.

[20] Purchase, H.C. and J. Hamer, Peer Review in Practice: eight years of experiences with Aropä. School of Computing Science University of Glasgow 31st January 2017.

[21] Cho, K. and C.D. Schunn, Scaffolded writing and rewriting in the discipline: A web-based reciprocal peer review system. Computers \& Education, 2007. 48(3): p. 409-426.

[22] Rice, W., Moodle E-Learning Course Development 2015: Packt Publishing Ltd.

[23] Sung, Y.-T., et al., The design and application of a webbased self-and peer-assessment system. Computers \& Education, 2005. 45(2): p. 187-202.

[24] Kali, Y. and M. Ronen. Design principles for online peer-evaluation: Fostering objectivity. in Proceedings of the 2005 conference on Computer support for collaborative learning: learning 2005: the next 10 years! 2005. International Society of the Learning Sciences.

[25] Paré, D.E. and S. Joordens, Peering into large lectures: examining peer and expert mark agreement using peerScholar, an online peer assessment tool. Journal of Computer Assisted Learning, 2008. 24(6): p. 526-540.

[26] Sluijsmans, D.M., et al., Peer assessment in problem based learning. Studies in educational evaluation, 2001. 27(2): p. 153-173.

[27] Honeychurch, S., et al., Peer assessment assisted by technology. International Journal of e-Assessment, 2013. $3(1)$.

[28] Leijen, D.A., A Novel Approach to Examine the Impact of Web-based Peer Review on the Revisions of L2 Writers. Computers and Composition, 2017. 43: p. 3554.

[29] Xiong, Y., et al. A proposed credibility index (CI) in peer assessment. in Poster presented at the annual meeting of the National Council on Measurement in Education, Philadelphia, PA. 2014.

[30] Goldin, I.M. and K.D. Ashley. Peering inside peer review with bayesian models. in Artificial Intelligence in Education. 2011. Springer.

[31] Edx. Open Response Assessments. 2017; Available from: https://edx.readthedocs.io/projects/open-edxbuilding-and-running-a-course/en/named-releasebirch/exercises_tools/open_response_assessments/index. html.

[32] Staubitz, T., et al. improving the peer assessment experience on MOOC platforms. in Proceedings of the Third (2016) ACM Conference on Learning@ Scale. 2016. ACM.

[33] IEEE, P1484.2/D7, 2000-11-28. Draft Standard for Learning Technology. Public and Private Information (PAPI) for Learners (PAPI Learner). Available at: 
http://ltsc.ieee.org/wg2/. Accessed on October 25, 2002. 2002.

[34] Oubahssi, L. and M. Grandbastien. From learner information packages to student models: Which continuum? in International Conference on Intelligent Tutoring Systems. 2006. Springer.

[35] Battou, A., Approche granulaire des objets pédagogiques en vue de l'adaptabilité dans le cadre des Environnements Informatiques pour l'Apprentissage Humain. 2012.

[36] Fini, A., The technological dimension of a massive open online course: The case of the CCK08 course tools. The International Review of Research in Open and Distributed Learning, 2009. 10(5).
[37] Brown, G.A., J. Bull, and M. Pendlebury, Assessing student learning in higher education. 2013: Routledge.

[38] Engelhard, G., Examining Rater Errors in the Assessment of Written Composition with a Many- Faceted Rasch Model. Journal of Educational Measurement, 1994. 31(2): p. 93-112.

[39] Felder, R.M. and L.K. Silverman, Learning and teaching styles in engineering education. Engineering education, 1988. 78(7): p. 674-681.

[40] Bellifemine, F.L., G. Caire, and D. Greenwood, Developing multi-agent systems with JADE. Vol. 7. 2007: John Wiley \& Sons. 